\title{
Clinical features and treatment outcomes of resected large cell neuroendocrine carcinoma of the lung
}

\author{
Jin Young Moon ${ }^{1}$, Seo Hee Choi ${ }^{1,2}$, Tae Hyung Kim ${ }^{1,3}$, Joongyo Lee ${ }^{1}$, Ji Hoon Pyo ${ }^{1}$, Yong Tae Kim¹, Seo Jin Lee ${ }^{1}$, \\ Hong In Yoon ${ }^{1}$, Jaeho Cho ${ }^{1}$, Chang Geol Lee ${ }^{1}$ \\ ${ }^{1}$ Department of Radiation Oncology, Yonsei Cancer Center, Yonsei University College of Medicine, Seoul, Korea \\ ${ }^{2}$ Department of Radiation Oncology, Yongin Severance Hospital, Yonsei University College of Medicine, Yongin, Korea \\ ${ }^{3}$ Department of Radiation Oncology, Nowon Eulji Medical Center, Eulji University School of Medicine, Seoul, Korea
}

Received: April 7, 2021

Revised: August 12, 2021

Accepted: August 23, 2021

\section{Correspondence: \\ Seo Hee Choi \\ Department of Radiation Oncology, Yongin Severance Hospital, Yonsei University College of Medicine, 363 Dongbaekjukjeon-daero, Giheung-gu, Yongin 16995, Korea. \\ Tel: +82-31-5189-8166 \\ E-mail: clickby_s@yuhs.ac ORCID: \\ https://orcid.org/0000-0002-4083-6414}

The abstract of this study was presented in a Poster Session at the Annual Meeting of the American Society for Radiation Oncology on October 24-28, 2020, in USA.
Purpose: Pulmonary large cell neuroendocrine carcinoma (LCNEC) is a high-grade lung neuroendocrine tumor with a poor prognosis, similar to small cell lung cancer (SCLC). However, it remains unclear whether to treat LCNEC as non-small-cell lung cancer (NSCLC) or as SCLC. We reviewed our experiences to suggest appropriate treatment strategy for resected pulmonary LCNEC.

Materials and Methods: Forty-four patients were treated for pathologically diagnosed pulmonary LCNEC during 2005-2018. We considered curative surgery first in early-stage or some locally advanced tumors, unless medically inoperable. Adjuvant treatments were decided considering patient's clinical and pathological features. After excluding two stage I tumors with radiotherapy alone and three stage III tumors with upfront chemotherapy, we analyzed 39 patients with stage I-III pulmonary LCNEC, who underwent curative resection first.

Results: Adjuvant chemotherapy (NSCLC-based 91\%, SCLC-based 9\%) was performed in 62\%, and adjuvant radiotherapy was done in three patients for pN2 or positive margin. None received prophylactic cranial irradiation (PCI). With a median follow-up of 30 months, the 2- and 5-year overall survival (OS) rates were $68 \%$ and $51 \%$, and the 2 - and 5-year recurrence-free survival (RFS) rates were $49 \%$ and $43 \%$, respectively. Aged $\geq 67$ years and SCLC-mixed pathology were significant poor prognostic factors for OS or RFS ( $p<0.05)$. Among 17 recurrences, regional failures were most common ( $=6)$, and there were five brain metastases.

Conclusions: Surgery and adjuvant treatment (without $\mathrm{PCI}$ ) could achieve favorable outcomes in pulmonary LCNEC, which was more similar to NSCLC, although some factors worsened the prognosis. The importance of intensified adjuvant therapies with multidisciplinary approach remains high.

Keywords: Neuroendocrine tumors, Lung neoplasms, Prognosis, Small cell lung carcinoma, Cranial irradiation

\section{Introduction}

Pulmonary large cell neuroendocrine carcinoma (LCNEC) is a rare malignancy of the lung, accounting for 1\%-3\% of all lung cancers [1]. Traditionally, LCNEC was recognized as a variant of large cell carcinoma, which is a type of non-small-cell lung cancer (NSCLC) in the World Health Organization (WHO) classification [2-4]. In the
2015 WHO classification, based on wider use of immunochemistry, it was re-classified as one of the neuroendocrine tumors along with three other main types: typical carcinoids, atypical carcinoids (lowgrade tumors), and small-cell lung cancer (SCLC) [5]. The current WHO classification defines LCNEC as a morphological NSCLC with histopathological features of neuroendocrine cancer and immunohistochemical expression of neuroendocrine markers. However, the 
survival prognosis is poor, with a high rate of lymph node involvement and distant metastasis, making it more similar to SCLC [4].

Due to its low incidence, and the consequent lack of relevant clinical trial data, understanding of the biological characteristics of pulmonary LCNEC is incomplete, and a standard of treatment has not yet been established. The primary recommended treatment for patients with early-stage, resectable pulmonary LCNEC is radical surgery (lobectomy or anatomic resection + mediastinal node dissection) [6]. Chemotherapy can be considered after surgery or instead of surgery in cases of advanced disease [7]. However, the most appropriate regimen (whether to follow the NSCLC-regimen or SCLC regimen) and the schedule of them are still not established, independently of the clinical stage of LCNEC [8]. Furthermore, the role of radiotherapy as post-operative treatment of local or advanced pulmonary LCNEC remains unclear, but some have suggested its use in the locally advanced disease setting $[9,10]$. Some researchers have recently proposed the need for prophylactic cranial irradiation (PCI) for this disease entity, based on its dismal prognosis and its high rate of brain metastasis, similar to SCLC, but a consensus has yet to be reached [10].

In this study, we reviewed our institutional treatment experience of pulmonary LCNEC, particularly in patients who underwent surgery and received adjuvant therapies after multidisciplinary discussion, with an objective to suggest the most appropriate direction for adjuvant treatment in each patient with resectable pulmonary LCNEC.

\section{Materials and Methods}

\section{Study design and patient selection}

First, 67 patients who were pathologically diagnosed with pulmonary LCNEC between January 2005 and December 2018 were retrospectively identified from our institutional database. Patients were included if they were diagnosed with this disease entity during this period and completed the entire session of the first treatment in our institution. Patients with distant metastasis at diagnosis ( $n=19$ ), without definite pathological confirmation of LCNEC ( $n=2)$, or who did not receive any cancer treatments any cancer treatments $(n=2)$ were excluded from this study. Finally, 44 patients with localized pulmonary LCNEC were identified. This study was approved by the Institutional Review Board of the Yonsei University Health System (No. 9-2021-0011) and the informed consent was waived.

\section{Treatment}

In our institution, treatment policies for patients with pulmonary LCNEC generally coincide with those for NSCLC. The TNM stage is classified based on the 8th edition of the American Joint Committee on Cancer (AJCC) cancer staging system. In early stage (I/II) tumors, as well as in some localized stage III tumors, surgery is considered first, unless there are medical contraindications. For tumors in a locally advanced stage, definitive concurrent chemoradiotherapy (CCRT) is considered first. After surgery, observation or adjuvant treatment comprising chemotherapy and/or radiotherapy is followed depending on pathological reports and stage. The chemotherapy regimen is usually planned according to treatment guidelines for NSCLC (e.g., platinum + gemcitabine/paclitaxel [TP] or platinum + vinorelbine [NP]). For radiotherapy, 45-54 Gy is prescribed for adjuvant cases, and 60-66 Gy is prescribed for definitive cases. Elective nodal irradiation or $\mathrm{PCl}$ is not usually performed in our institution.

\section{Statistical analyses}

Overall survival (OS) and recurrence-free survival (RFS) were calculated from the date of pathological diagnosis of pulmonary LCNEC to the date for each end point. Locoregional failure was defined as recurrence in the same lung or lymph nodes in the hilum, mediastinum, or supraclavicular fossa. Distant failure was defined as failure at another site. Brain metastasis-free survival was defined as the interval between the date of pathological diagnosis and the date of an imaging study (brain magnetic resonance imaging [MRI]) showing evidence of brain metastasis. To compare the brain metastasis rate of LCNEC with that of SCLC, patients diagnosed as limited stage SCLC during the same period in our institution were selected as a control group. We estimated the brain metastasis-free survival rate of patients with LCNEC and patients with $\operatorname{SCLC~}( \pm \mathrm{PCI})$, and compared the survival rates to evaluate the need for $\mathrm{PCl}$ in $\mathrm{LCNEC}$ patients. Survival outcomes were analyzed using the Kaplan-Meier method and log-rank tests. Univariate analysis and multivariate analysis were performed by using Cox regression models. p-values $<0.05$ were considered significant. Statistical analyses were performed using SPSS statistical software version 25.0 (IBM Corp., Armonk, NY, USA).

\section{Results}

\section{Patient and treatment characteristics}

Of the 44 patients with pathologic diagnosed and received curative treatments in our institution, 20 patients had stage I, 13 had stage II, and 11 had stage III disease. As our institutional policy, surgical resection was indicated when feasible for each patient with localized LCNEC. Among the 20 patients with stage I tumors, only two patients received definitive radiotherapy alone due to inoperable medical conditions. Among 13 patients with stage II tumors, all re- 
ceived upfront surgery ( \pm adjuvant therapy). Among 11 patients with stage III tumors, three patients received definitive CCRT for $\mathrm{N} 3$ stage node metastases. The treatment flow diagram is shown in Fig. 1. Thus, 39 patients with stage I-III $\operatorname{LCNEC~}(\mid=18$, II = 13, III $=8$ ) first underwent curative resection of the primary tumor; the treatment outcomes of these patients were analyzed in this study.

Among 44 patients with localized pulmonary LCNEC, 39 patients who underwent curative tumor resection first were included in this study. Baseline clinical characteristics of patients and tumors, and treatment data are shown in Table 1. According to the AJCC cancer staging system, there were 18,13 , and eight patients with tumors at stage I, II, or III, respectively. Most patients (36/39) underwent primary lobectomy with or without mediastinal lymph node dissection, while two underwent wedge resection, and one underwent pneumonectomy. A clear resection margin was achieved in 35 (90\%) patients. Among four patients with R1-resected disease, two patients received adjuvant chemotherapy only, one received adjuvant chemotherapy and radiotherapy sequentially (50 Gy in 25 fractions), and one received adjuvant CCRT (51.6 Gy in 24 fractions).

In other R0-resected patients, whether to add adjuvant treatments or which regimen to choose were determined considering the patients' condition and tumor stage after multidisciplinary discussion. For patients with stage IA tumors (pT1N0, $n=12$ ), most ( $n$ $=10 ; 83 \%$ ) did not receive any adjuvant therapy, while $67 \%$ of patients with stage IB tumors ( $\mathrm{pT} 2 \mathrm{NO}, \mathrm{n}=6$ ) received adjuvant chemotherapy (all NSCLC-based treatment). In patients with stage
IIA $(n=1)$ or IIB $(n=12)$ tumors, most patients ( $n=11 ; 85 \%)$ received adjuvant chemotherapy, which mostly consisted of an NSCLC-based regimen, excluding two patients in whom an SCLCbased EP regimen was used. In patients with stage IIIA $(n=7)$ or IIIB $(n=1)$ tumors, all except one received adjuvant therapy. Adjuvant chemotherapy with or without sequential radiotherapy was performed in six patients with stage IIIA tumors, and adjuvant CCRT was given to one patient with stage IIIB disease. The chemotherapy regimen was all NSCLC-based for stage IIIA disease, and was SCLC-based for stage IIB disease. One patient did not receive any adjuvant therapy for a clearly resected tumor at pT3N1 (IIIA) stage, because cytotoxic chemotherapy was intolerable due to the patient's poor condition. This patient experienced early recurrence only 4 months after surgery. Adjuvant radiotherapy was performed in three patients with stage III tumors, and a median dose of $50 \mathrm{~Gy}$ (range, 45 to $54 \mathrm{~Gy}$ ) was delivered for pN2 disease $(n=1)$ or a positive resection margin $(n=2)$. No patient received $\mathrm{PCl}$. Details of adjuvant therapy according to the tumor stage are shown in Fig. 1.

\section{Survival outcomes and prognostic factors}

The median follow-up time was 30 months (range, 2 to 161 months) from the first diagnosis. In all patients, the 2- and 5-year OS rates were $67.5 \%$ and $51.1 \%$, and the 2- and 5-year RFS rates were $49.4 \%$ and $42.9 \%$, respectively (Fig. 2A). Two-year OS rates were $76.5 \%, 57.5 \%$, and $62.5 \%$ in stage I, stage II, and stage III, respectively (Fig. 2B), while the 2-year RFS rates were $76.5 \%$,
Localized pulmonary LCNEC

(2005-2018, n=44)

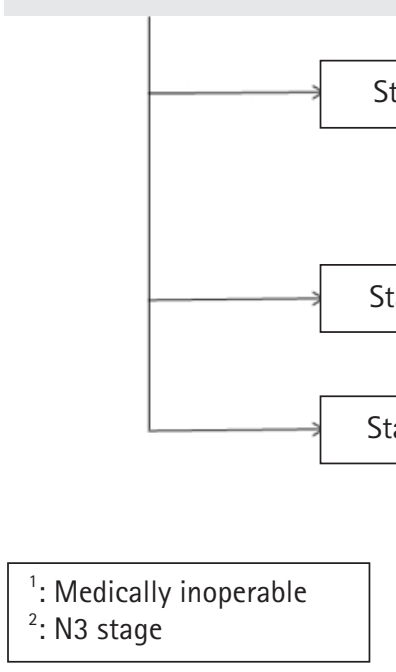

Institutional policy for pulmonary LCNEC

: Usually determined following NSCLC's

- Resectable tumors: Upfront surgery

- Unresectable tumors: RT or CCRT

- Adjuvant therapies: In high risk patients (NSCLC regimen-preferred)

$\rightarrow$ 'Definitive RT $(\mathrm{n}=2)$

$\rightarrow$ Op only $(n=12)$

$O p+$ Adjuvant CTx $(n=6)$

Op only $(n=2)$

$\rightarrow$ Op + Adjuvant CTx $(n=11)$

$\longrightarrow 0 p+$ Adjuvant CTx $(n=4)$

Op + Adjuvant CCRT ( $n=3)$

Op only $(n=1)$

${ }^{2}$ Definitive CCRT or CTx $(n=3)$

Fig. 1. Treatment flow diagram for eligible patients with localized pulmonary large cell neuroendocrine carcinoma. LCNEC, large cell neuroendocrine carcinoma; NSCLC, non-small-cell lung cancer; RT, radiotherapy; CCRT, concurrent chemo-radiotherapy; CTx, chemotherapy. 
Table 1. Patient and tumor characteristics

\begin{tabular}{|c|c|}
\hline Characteristic & Value \\
\hline \multicolumn{2}{|l|}{ Sex } \\
\hline Male & $35(90)$ \\
\hline Female & 4 (10) \\
\hline Age $(y r)$ & $67(46-74)$ \\
\hline \multicolumn{2}{|l|}{ Smoking history } \\
\hline Yes & $28(72)$ \\
\hline No & $11(28)$ \\
\hline \multicolumn{2}{|l|}{ T stage } \\
\hline $\mathrm{T} 1$ & $17(43)$ \\
\hline $\mathrm{T} 2$ & $14(36)$ \\
\hline T3 & $8(21)$ \\
\hline $\mathrm{T} 4$ & $0(0)$ \\
\hline \multicolumn{2}{|l|}{ N stage } \\
\hline No & $25(64)$ \\
\hline N1 & $7(18)$ \\
\hline N2 & $7(18)$ \\
\hline N3 & $0(0)$ \\
\hline \multicolumn{2}{|l|}{ AJCC stage } \\
\hline $\mathrm{IA} / \mathrm{IB}$ & $18(46)$ \\
\hline$\|A /\| B$ & $13(33)$ \\
\hline IIIA/IIIB & $8(21)$ \\
\hline \multicolumn{2}{|c|}{ Surgery (primary thoracic surgery) } \\
\hline Wedge resection & $2(5)$ \\
\hline Lobectomy & $36(92)$ \\
\hline Pneumonectomy & $1(3)$ \\
\hline \multicolumn{2}{|l|}{ Histology } \\
\hline Pure LCNEC & $30(77)$ \\
\hline NSCLC-mixed & 7 (18) \\
\hline SCLC-mixed & $2(5)$ \\
\hline Lymphovascular invasion & $20(51)$ \\
\hline Perineural invasion & $3(8)$ \\
\hline \multicolumn{2}{|l|}{ Resection status } \\
\hline Ro & $35(90)$ \\
\hline $\mathrm{R} 1$ & 4 (10) \\
\hline R2 & $0(0)$ \\
\hline Adjuvant radiotherapy & $3(8)$ \\
\hline Dose (Gy) & $50(45-54)$ \\
\hline \multicolumn{2}{|l|}{ Radiotherapy technique } \\
\hline 3D-CRT & 2 \\
\hline IMRT & 1 \\
\hline Adjuvant chemotherapy & $24(62)$ \\
\hline \multicolumn{2}{|l|}{ Chemotherapy regimen } \\
\hline NSCLC-based & $21(53)$ \\
\hline Navelbine + Cisplatin & 19 \\
\hline Genexol + Neoplatin & 2 \\
\hline SCLC-based & $3(8)$ \\
\hline Etoposide + Cisplatin & 3 \\
\hline
\end{tabular}

Values are presented as number (\%) or median (range). AJCC, American Joint Committee on Cancer; LCNEC, large cell neuroendocrine carcinoma; NSCLC, non-small cell lung cancer; SCLC, small cell lung cancer; 3D-CRT, three-dimensional conformal radiotherapy; IMRT, intensity-modulated radiotherapy.
57.5\%, and 62.5\%, respectively (Fig. 2C).

In both univariate and multivariate analyses for $0 S_{\text {, age }}(\geq 67$ years) and pathological subtype (SCLC-mixed) were identified as independently significant prognostic factors $(p=0.036$; hazard ratio $[\mathrm{HR}]=2.805 ; 95 \%$ confidence interval $[\mathrm{Cl}], 1.068-7.365$ and $p=$ $0.003 ; \mathrm{HR}=16.125 ; 95 \% \mathrm{Cl}$, 2.615-99.434; respectively) (Table 2). In patients aged $\geq 67$ years, the 2 - and 5 -year OS rates were 53.6\% and 21.4\%, respectively (Fig. 3A). Patients with LCNEC mixed with SCLC $(n=2)$ died within a year, while 1-year OS rates in patients with pure LCNEC $(n=30)$ and LCNEC mixed with NSCLC ( $n=7$ ) were 93.1\% and 85.7\%, respectively (Fig. 3B). Two patients (pT3N0 and pT3N2) had SCLC-mixed type tumors, and both received EP chemotherapy. They died at a median of 7.4 months: one due to encephalitis during adjuvant CCRT and another due to interstitial lung disease that was aggravated after four cycles of chemotherapy. In univariate and multivariate analyses for RFS, pathological subtype was also the only significant factor $(\mathrm{p}=$ $0.033 ; \mathrm{HR}=5.533 ; 95 \% \mathrm{Cl}, 1.150-26.615)$.

\section{Pattern of failure}

Disease recurrence was observed in 17 (43\%) patients at a median of 11.5 months (range, 2 to 111 months) after diagnosis: seven patients (35\%) with stage I tumors, seven patients (54\%) with stage II tumors, and three patients (27\%) with stage III tumors. Most cases were locoregional failures $(13 / 17 ; 76 \%)$ with five simultaneously diagnosed distant failures. Among eight isolated locoregional failures, regional failures were more common $(n=6)$ than local failures $(n=2)$. Among four isolated distant failures, three patients experienced brain metastases as a first failure, without any intra-thoracic recurrences (Supplementary Fig. S1).

For the location of locoregional failures, only two cases (15\%) recurred within the resection margin or in the same lobe of the lung, while the other 11 cases (85\%) were regional node metastasis. The most common site of regional failures was mediastinal lymph nodes ( $\mathrm{n}=7$ ), followed by supraclavicular fossa lymph nodes ( $n=5)$ and hilar lymph nodes ( $n=5)$. All except one patient had received salvage treatment, but only two are currently alive with disease. Details of patients who underwent any recurrence are shown in Table 3.

\section{Brain metastasis-free survival}

The 2- and 5-year brain metastasis-free survival rates were $65.1 \%$ and $46.3 \%$, respectively, and median survival was 58.8 months. There were five cases of brain metastases that developed as first failure with $(n=2)$ or without $(n=3)$ other types of recurrence, and one case that developed 5 months after isolated locoregional recurrence. The rate of brain metastasis development was 15\% in 

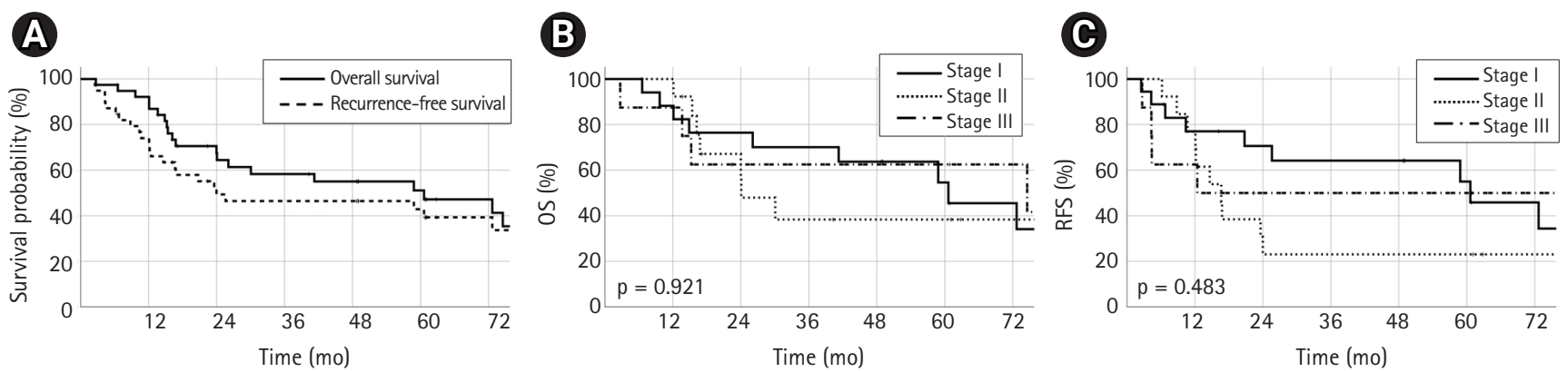

Fig. 2. (A) Kaplan-Meier curves for overall survival (OS) and recurrence-free survival (RFS) in all patients, (B) Kaplan-Meier curves for OS rate based on tumor stage, and (C) RFS rate based on tumor stage.
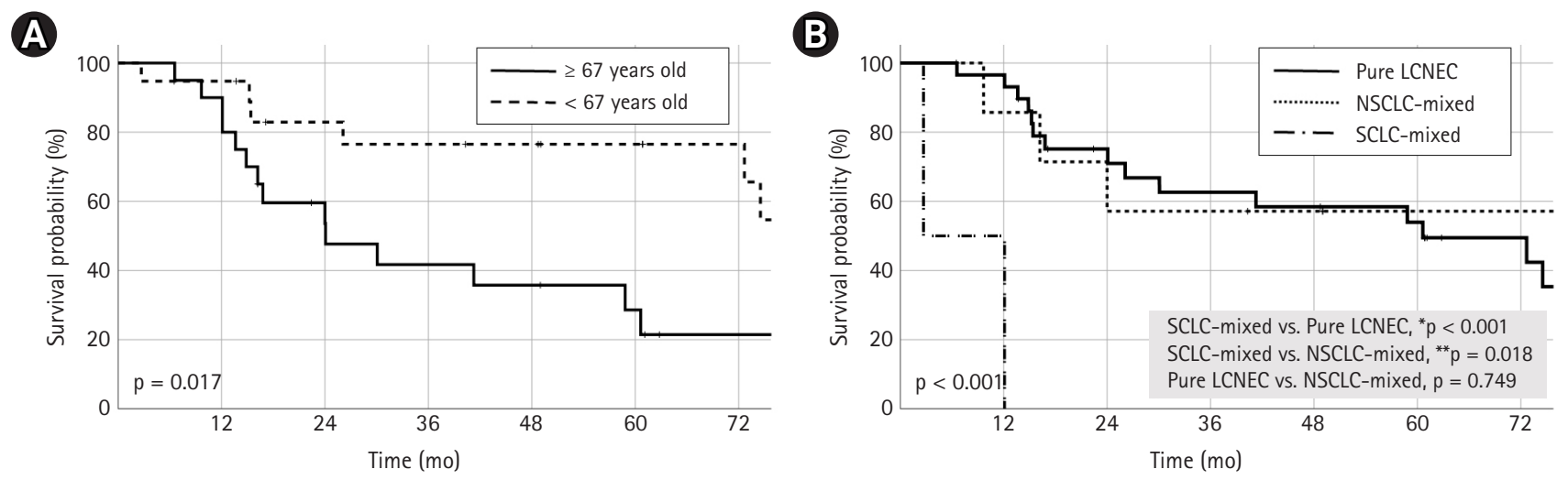

Fig. 3. Kaplan-Meier curves for overall survival stratified according to (A) age and (B) pathological subtype. Patients' age is divided into $\geq 67$ years and $<67$ years, and pathological subtype is divided into three: pure LCNEC, LCNEC mixed with NSCLC, and LCNEC mixed with SCLC. LCNEC, large cell neuroendocrine carcinoma; NSCLC, non-small-cell lung cancer; SCLC, small-cell lung cancer.

Table 2. Prognostic factors for overall survivals in patients with resected pulmonary LCNEC

\begin{tabular}{|c|c|c|c|c|}
\hline & \multicolumn{2}{|c|}{ Univariate analysis } & \multicolumn{2}{|c|}{ Multivariate analysis } \\
\hline & $\mathrm{HR}(95 \% \mathrm{Cl})$ & p-value & $\mathrm{HR}(95 \% \mathrm{Cl})$ & $\mathrm{p}$-value \\
\hline Age ( $\geq 67 \mathrm{yr})$ & $2.992(1.166-7.678)$ & $0.023^{*}$ & $2.805(1.068-7.365)$ & $0.036^{*}$ \\
\hline Sex (male) & $0.483(0.064-3.617)$ & 0.479 & - & \\
\hline Stage I & - & 0.78 & - & \\
\hline Stage II & $1.254(0.473-3.327)$ & 0.649 & - & \\
\hline Stage III & $0.812(0.253-2.602)$ & 0.726 & - & \\
\hline Adjuvant chemotherapy & $0.962(0.397-2.329)$ & 0.932 & - & \\
\hline Mixed pathology (pure LCNEC) & - & $0.005^{*}$ & - & $0.011^{*}$ \\
\hline Mixed with NSCLC & 1.193 (0.391-3.638) & 0.756 & $1.348(0.439-4.144)$ & 0.602 \\
\hline Mixed with SCLC & 21.061 (3.414-129.935) & $0.001^{*}$ & $16.125(2.615-99.434)$ & 0.003 \\
\hline
\end{tabular}

LCNEC, large cell neuroendocrine carcinoma; NSCLC, non-small cell lung cancer; SCLC, small cell lung cancer; $\mathrm{HR}$, hazard ration; Cl, confidence interval.

${ }^{*} p<0.05$.

all patients, and was significantly higher in stage II or III than in stage I tumors-stage I, 6\% (1/18); stage II, 23\% (3/13); stage III, $25 \%(2 / 8)$. In these six patients, brain metastases developed at a median of 8.5 months after the first diagnosis. In the three cases in which brain metastasis occurred as an isolated first failure, the interval was a median of 6.2 months, which was shorter than in the rest of the patients (median 10.8 months). However, with salvage treatments, including surgery and adjuvant radiotherapy, the survival time of these patients after the diagnosis of brain metastasis was longer than that of the rest of the patients (median 7.5 vs. 5.4 months) (Table 3).

The brain metastasis rate between LCNEC and corresponding 


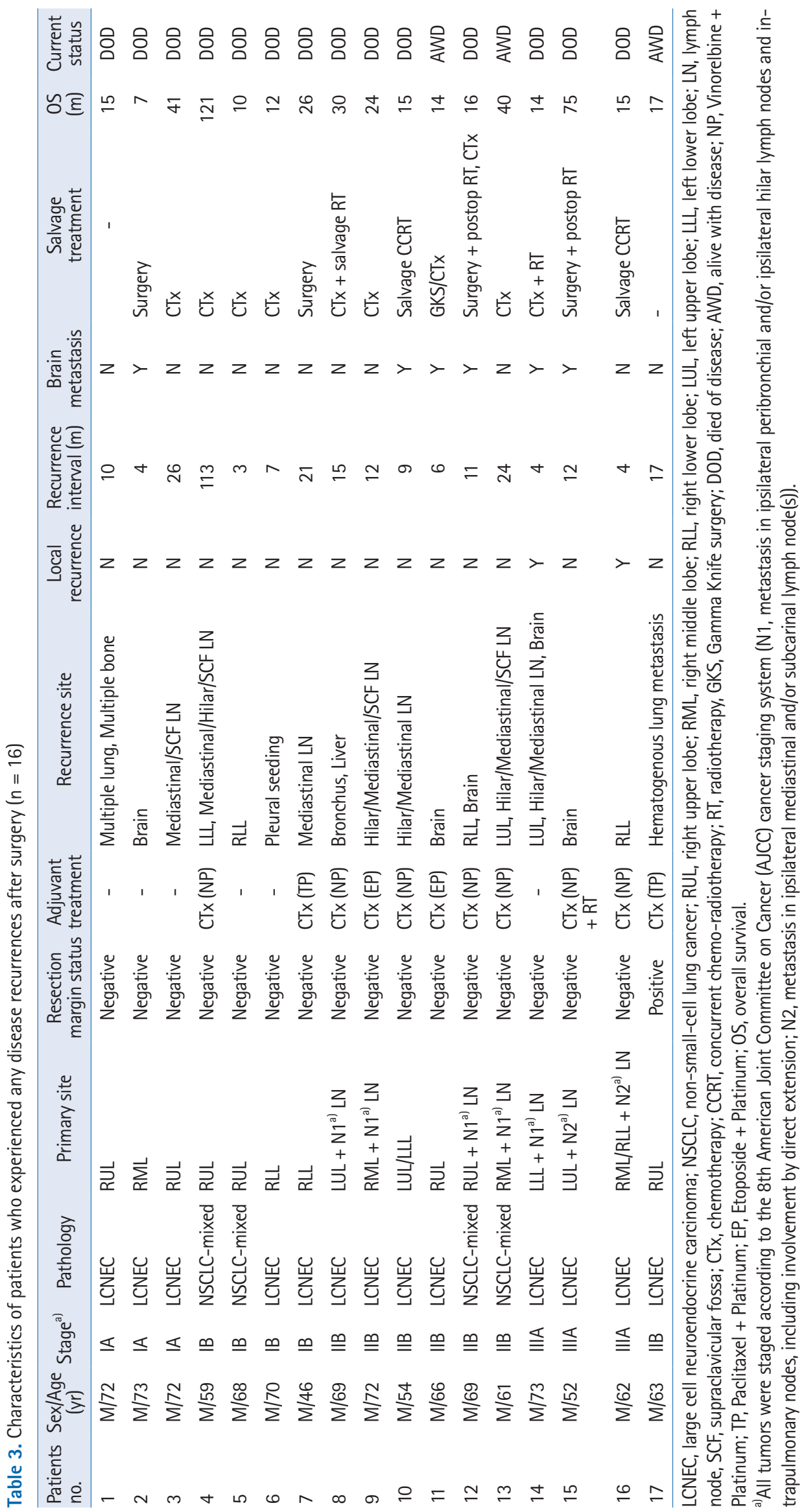


SCLC cohorts are available in Supplementary Table S1 and Fig. S2. The overall brain recurrence rates were $15.4 \%$ (LCENC cohort, $n=$ 39) and $24.1 \%$ (SCLC cohort, $n=108$ ). The 2-year and 5-year brain metastasis-free survival rates were $62.1 \%$ and $46.3 \%$ and $51.2 \%$ and $21.4 \%$, respectively. The brain metastasis-free survival rate was significantly better in the LCNEC cohort than in the SCLC cohorts ( $p=0.028$ ). In subgroup analysis, the brain metastasis-free survival rate was significantly better in LCNEC patients than in SCLC patients with or without PCI ( $p=0.028$ ) (LCNEC vs. SCLC without PCl, $p=0.010$; LCNEC vs. SCLC with PCl, $p=0.130$; and $\mathrm{SCLC}$ with $\mathrm{PCl}$ vs. SCLC without $\mathrm{PCl}, \mathrm{p}=0.068$ ) (Supplementary Fig. S2).

\section{Discussion and Conclusion}

In this study, we achieved favorable survival outcomes with surgical resection and appropriate adjuvant therapies in keeping with NSCLC protocols. Survival outcomes were comparable or better than those of previous studies $[4,9,11,12]$ (Table 4). Despite smaller study populations, some factors, such as older age and mixed histology, were significantly associated with prognosis. Brain metastasis rate and brain metastasis-free survival rate were significantly better than those for SCLC, with or without PCl. Notwithstanding, older age, primary location (main bronchus), and no adjuvant chemotherapy have been shown to exhibit significant associations with poor prognosis in previous studies $[7,10]$.

LCNEC has been regarded as a disease entity that shows features of both NSCLC and SCLC. As reported in SEER data, the 1-, 2-, and 4 -year OS rate in patients with resectable pulmonary LCNEC was $76 \%, 56 \%$, and $41 \%$, respectively, which were similar to that of other types of large cell carcinoma and was different from SCLC [4]. In our study, the 2- and 5-year OS rates were 68\% and 51\%, respectively, which were slightly better than that reported before. According to recent studies, the clinical presentation of LCNEC appears to be similar to that of another high-grade neuroendocrine pulmonary tumor, SCLC, with some exceptions. Primary LCNECs tend to be located peripherally rather than centrally, and presenta-

Table 4. Comparison of overall survival rates with previous studies

\begin{tabular}{lcccc}
\hline & \multicolumn{4}{c}{ Overall survival (\%) } \\
\cline { 2 - 5 } & $1 \mathrm{yr}$ & $2 \mathrm{yr}$ & $3 \mathrm{yr}$ & $5 \mathrm{yr}$ \\
\hline Our study & 92.2 & 67.5 & 58.3 & 51.1 \\
SEER database [11] & 74.5 & 55.8 & 46.7 & NA \\
Jin et al. [9] & $\mathrm{NA}$ & $\mathrm{NA}$ & 50.7 & 41.2 \\
Varlotto et al. [4] (pT1N0) & 90 & 76 & 60 & NA \\
Kinslow et al. [12] & $\mathrm{NA}$ & 61 & $\mathrm{NA}$ & $\mathrm{NA}$ \\
\hline
\end{tabular}

SEER, Surveillance, Epidemiology, and End Results; NA, not available. tion of LCNECs with early-stage (I/II) disease is more common than for SCLC (25\% vs. <5\%) [13]. Thus, patients with LCNEC more commonly undergo resection. In the US SEER database [4], clinical, histopathological, and biological features as well as survival rates of patients with resected LCNEC are more similar to those of other large cell carcinomas than to those of SCLC. On the other hand, according to recent population-based studies [12,14], it has been suggested that LCNEC, particularly in the advanced stage, may behave more similarly to SCLC. These observations should be taken into account when deciding on a treatment regimen.

Due to the rarity of LCNEC, there are no large randomized trials that define an optimal treatment approach [15]. Treatment recommendations are based on extrapolation from approaches used in patients with SCLC and NSCLC and the established literature, which is primarily retrospective in nature. Surgical resection is usually indicated, when feasible, for patients with early-stage LCNEC, and CCRT followed by additional cycles of chemotherapy can be considered, similar to limited stage SCLC, for patients with positive mediastinal nodes after resection and for those with unresectable stage III disease. Because of poor prognosis after surgery, extensive adjuvant therapies may be indicated in LCNEC. While additional adjuvant chemotherapy might contribute to improved survival [15-19], the role of adjuvant radiotherapy remains unclear [9]. Optimal systemic treatment has not been adequately established [20]. Moreover, in the American Society of Clinical Oncology guidelines, either the SCLC regimen or the same regimen as for NSCLC is advised for LCNEC. However, SCLC-type chemotherapy is considered by expert opinion to be most appropriate [21], as LCNEC appears to respond similarly to SCLC according to several observational and genomic studies [22-24]. Notwithstanding, recent studies have reported a low response rate or higher resistance to SCLC-type chemotherapy [1,25-28]. To date, with no standard regimen, although SCLC regimens can be considered in advanced diseases, NSCLC regimens may also be reasonable alternatives.

Patterns of failure in our patients were distinguishable from those of NSCLC $[29,30]$. Regarding recurrence location, a few cases $(13 \%)$ involved recurrence at resection margins or the same lobe of the lung. A high rate of recurrence (approximately 50\%) occurred at the surgical stump in NSCLC, even though all of the patients had a clear resection margin. All cases of regional lymph node recurrence included the mediastinal lymph node area (with or without hilar lymph nodes), and supraclavicular lymph node recurrence was also more common than that in NSCLC (29.4\% vs. 8\%-10\%). Contralateral lung metastasis, distant metastasis, and brain metastasis even as a first recurrence were common in our LCNEC cohort. Our results for patterns of failure in LCNEC patients highlight difficulties with salvage treatments and the need for intensified adjuvant 
treatment strategies, even after clear resection.

A significant difference in the management of LCNEC and SCLC is that $\mathrm{PCl}$ is recommended for many patients with $\mathrm{SCLC}$, but not for those with LCNEC. Multiple large meta-analyses have shown an increase in survival as well as a decrease in brain metastases in patients with limited-stage SCLC receiving $\mathrm{PCl}$. However, there are still controversies about the application of $\mathrm{PCl}$ for pulmonary LCNEC [15]. In our study, the rate of brain metastasis was 15\%, and was higher for stage II/III tumors than for stage I disease. It was lower than that previously reported for patients with SCLC [31]. Even in our sub-analyses, the brain metastasis-free survival rate was significantly higher in LCNEC patients than in SCLC patients, regardless of the use of $\mathrm{PCl}(\mathrm{p}=0.028)$. On the other hand, in other population studies, the rates of brain metastases were higher in LCNEC than in SCLC (increasing in stage IV disease), suggesting the need for $\mathrm{PCl}$ in LCNEC. One possible contribution to this discrepancy is that our study only included relatively early-stage tumors for which surgery was indicated. Therefore, the brain metastasis rate was low, and the metastatic brain lesions could be treated locally, without a critical effect on prognosis.

There were some limitations to our study. Although our patients were followed-up for a long period, the number of patients was insufficient to arrive at a definite conclusion. In particular, there have been some discrepancies in the chemotherapy regimen in our institution. Except that the SCLC-based regimen was selected in a few patients with SCLC-mixed pathology, the NSCLC-based regimen was generally chosen. Second, the prognosis of stage II and III disease were reversed in some points. This might be due to the exclusion of stage III patients who had supraclavicular lymph nodes at diagnosis (and were thus expected to show a poor prognosis) and received CCRT instead of surgery. A follow-up study of a larger number of patients would be helpful.

Despite these limitations, our study had several strengths. In our study, the 2- and 5-year OS rates were favorable, compared to available data. This may be due to curative resection of localized pulmonary LCNEC and sufficient adjuvant therapies after multidisciplinary discussion. Furthermore, our data suggest that patients with mixed histology with SCLC are expected to have poor prognosis. In general, a pathology indicating combined SCLC has been reported to show a poor prognosis, similar to SCLC, as well as chemo-resistance [32]. Also, SCLC-based chemotherapy might be too toxic without contributing to survival improvement. Although more studies including a larger number of patients are needed, our findings suggest that SCLC-based chemotherapy might not necessarily be a good alternative for pulmonary LCNEC.

In conclusion, treatment outcomes for patients with LCNEC that underwent surgical resection were more favorable than those re- ported in historical data of limited stage SCLC and similar to those of NSCLC. However, the importance of using intensified adjuvant therapies remains high, because failure patterns mostly involved regional failure or distant metastasis at the first recurrence, rather than local failure. New chemotherapy regimens or mediastinal radiotherapy might be alternatives, although SCLC-type chemotherapy remains questionable, according to our results. Early application of $\mathrm{PCl}$ did not appear to be necessary. Although further studies in a larger number of patients with resectable, as well as unresectable LCNEC, are warranted, our findings yield insight into suitable treatment approaches for LCNEC.

\section{Conflict of Interest}

No potential conflict of interest relevant to this article was reported.

\section{Acknowledgements}

This study was supported by a faculty research grant of Yonsei University College of Medicine for (No. 6-2020-0115).

\section{Supplementary Materials}

Supplementary materials can be found via https://doi.org/10.3857/ roj.2021.00423.

\section{References}

1. Fasano M, Della Corte CM, Papaccio F, Ciardiello F, Morgillo F. Pulmonary large-cell neuroendocrine carcinoma: from epidemiology to therapy. J Thorac Oncol 2015;10:1133-41.

2. Brambilla $E_{1}$ Travis WD, Colby TV, Corrin B, Shimosato Y. The new World Health Organization classification of lung tumours. Eur Respir J 2001;18:1059-68.

3. Beasley MB, Brambilla E, Travis WD. The 2004 World Health Organization classification of lung tumors. Semin Roentgenol 2005;40:90-7.

4. Varlotto JM, Medford-Davis LN, Recht A, et al. Should large cell neuroendocrine lung carcinoma be classified and treated as a small cell lung cancer or with other large cell carcinomas? J Thorac Oncol 2011;6:1050-8.

5. Travis WD, Brambilla E, Burke AP, Marx A, Nicholson AG. Introduction to the 2015 World Health Organization classification of tumors of the lung, pleura, thymus, and heart. J Thorac Oncol 2015;10:1240-2.

6. Shah MH, Goldner WS, Halfdanarson TR, et al. NCCN guidelines 
insights: neuroendocrine and adrenal tumors, version 2.2018. J Natl Compr Canc Netw 2018;16:693-702.

7. Gu J, Gong D, Wang Y, et al. The demographic and treatment options for patients with large cell neuroendocrine carcinoma of the lung. Cancer Med 2019;8:2979-93.

8. Rossi G, Cavazza A, Marchioni A, et al. Role of chemotherapy and the receptor tyrosine kinases KIT, PDGFRalpha, PDGFRbeta, and Met in large-cell neuroendocrine carcinoma of the lung. J Clin Oncol 2005;23:8774-85.

9. Jin G, Xu L, Song X. The role of postoperative radiotherapy (PORT) in pulmonary large cell neuroendocrine carcinoma (PLCNEC). Cancer Radiother 2020;24:215-21.

10. Cao $L$, Wu HF, Zhao $L$, et al. The role of radiotherapy in pulmonary large cell neuroendocrine carcinoma: propensity score matching analysis. J Radiat Res 2020;61:594-601.

11. Wang J, Ye L, Cai H, Jin M. Comparative study of large cell neuroendocrine carcinoma and small cell lung carcinoma in highgrade neuroendocrine tumors of the lung: a large population-based study. J Cancer 2019;10:4226-36.

12. Kinslow CJ, May MS, Saqi A, et al. Large-cell neuroendocrine carcinoma of the lung: a population-based study. Clin Lung Cancer 2020;21:e99-113.

13. Lo Russo G, Pusceddu S, Proto $C_{\text {, }}$ et al. Treatment of lung large cell neuroendocrine carcinoma. Tumour Biol 2016;37:7047-57.

14. Derks JL, Hendriks LE, Buikhuisen WA, et al. Clinical features of large cell neuroendocrine carcinoma: a population-based overview. Eur Respir J 2016;47:615-24.

15. Glisson BS, Moran CA. Large-cell neuroendocrine carcinoma: controversies in diagnosis and treatment. J Natl Compr Canc Netw 2011;9:1122-9.

16. Veronesi G, Morandi U, Alloisio M, et al. Large cell neuroendocrine carcinoma of the lung: a retrospective analysis of 144 surgical cases. Lung Cancer 2006;53:111-5.

17. Iyoda A, Hiroshima K, Moriya Y, et al. Prospective study of adjuvant chemotherapy for pulmonary large cell neuroendocrine carcinoma. Ann Thorac Surg 2006;82:1802-7.

18. Iyoda A, Hiroshima K, Moriya Y, et al. Postoperative recurrence and the role of adjuvant chemotherapy in patients with pulmonary large-cell neuroendocrine carcinoma. J Thorac Cardiovasc Surg 2009;138:446-53.

19. Raman V, Jawitz OK, Yang CJ, et al. Adjuvant therapy for patients with early large cell lung neuroendocrine cancer: a national analysis. Ann Thorac Surg 2019;108:377-83.

20. Reck M, Popat S, Reinmuth N, et al. Metastatic non-small-cell lung cancer (NSCLC): ESMO Clinical Practice Guidelines for diagnosis, treatment and follow-up. Ann Oncol 2014;25 Suppl 3:iii27-39.

21. Hanna N, Johnson D, Temin S, et al. Systemic therapy for stage IV non-small-cell lung cancer: American Society of Clinical Oncology Clinical Practice Guideline update. J Clin Oncol 2017;35: 3484-515.

22. George J, Lim JS, Jang SJ, et al. Comprehensive genomic profiles of small cell lung cancer. Nature 2015;524:47-53.

23. Rekhtman N, Pietanza MC, Hellmann MD, et al. Next-generation sequencing of pulmonary large cell neuroendocrine carcinoma reveals small cell carcinoma-like and non-small cell carcinoma-like subsets. Clin Cancer Res 2016;22:3618-29.

24. Asamura H, Kameya T, Matsuno Y, et al. Neuroendocrine neoplasms of the lung: a prognostic spectrum. J Clin Oncol 2006;24:70-6.

25. Le Treut J, Sault MC, Lena H, et al. Multicentre phase II study of cisplatin-etoposide chemotherapy for advanced large-cell neuroendocrine lung carcinoma: the GFPC 0302 study. Ann Oncol 2013;24:1548-52.

26. Niho S, Kenmotsu H, Sekine I, et al. Combination chemotherapy with irinotecan and cisplatin for large-cell neuroendocrine carcinoma of the lung: a multicenter phase II study. J Thorac Oncol 2013;8:980-4.

27. Rossi A, Di Maio M, Chiodini $P$, et al. Carboplatin- or cisplatin-based chemotherapy in first-line treatment of small-cell lung cancer: the COCIS meta-analysis of individual patient data. J Clin Oncol 2012;30:1692-8.

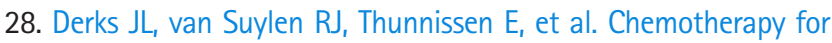
pulmonary large cell neuroendocrine carcinomas: does the regimen matter? Eur Respir J 2017;49:1601838.

29. Kelsey $C R$, Light $K L$, Marks LB. Patterns of failure after resection of non-small-cell lung cancer: implications for postoperative radiation therapy volumes. Int J Radiat Oncol Biol Phys 2006;65: 1097-105.

30. Feng $W_{1}$ Fu $X L$, Cai XW, et al. Patterns of local-regional failure in completely resected stage IIIA(N2) non-small cell lung cancer cases: implications for postoperative radiation therapy clinical target volume design. Int J Radiat Oncol Biol Phys 2014;88: 1100-7.

31. Lukas RV, Gondi V, Kamson DO, Kumthekar P, Salgia R. State-ofthe-art considerations in small cell lung cancer brain metastases. Oncotarget 2017;8:71223-33.

32. Qin J, Lu H. Combined small-cell lung carcinoma. Onco Targets Ther 2018;11:3505-11. 